\title{
An Investigation of the Drivers of Social Commerce and e-Word-of-Mouth Intentions: Elucidating the Role of Social Commerce in E-business
}

M. Awais Shakir Goraya, Zhu Jing, Mahmud Akhter Shareef, Muhammad Imran, Aneela Malik and M. Shakaib Akram

\begin{abstract}
Building on social commerce (s-commerce) perspectives and the trust transfer theory, this study develops a theoretical model that explains the indirect effects of two types of s-commerce attributes (community and platform) on behavioral outcomes (s-commerce intentions and e-Word-of-Mouth (e-WOM) intentions) through trust in community and platform. We analyze data collected from s-commerce users on travel booking websites using structural equation modeling technique. Results confirm that s-commerce intentions and e-WOM intentions are contingent upon s-commerce community and platform attributes. Moreover, the results provide evidence for the mediating effects of trust in community and platform on the relationship between s-commerce attributes and behavioral outcomes. The study provides further insights about the impact of scommerce experience on s-commerce intention and e-WOM intention. Moreover, this study contributes to s-commerce research and practice by developing and validating the role of s-commerce community and platform attributes in forming consumers' scommerce behavioral outcomes.
\end{abstract}

Keywords: Social commerce, community attributes, platform attributes, trust in platform, trust in community, social commerce intention, e-WOM intention. 


\section{Introduction}

Social commerce (s-commerce), generally known as social business, bears no specificity regarding its academic definition as it carries various connotations in academic insights (Liang, Ho, Li, \& Turban, 2011). However, the extant literature presents the definition of s-commerce as "the use of Internet-based media which enable users to participate in the selling, buying, comparing, and sharing of information about products and services in an online marketplace and communities" (Busalim \& Hussin, 2016). The concept of scommerce was formed in 2005, and ever since it has evolved through academic acumens and market trends. S-commerce differentiates itself from e-commerce by providing more sales/communication avenues to consumers and organizations in the current Internet era. In e-commerce business models, consumers are independent of each other when making their purchase decision, however, in a s-commerce setting consumers have a collaborative and connected relationship (Zhou, Zhang, \& Zimmermannc, 2013). While scommerce is convincingly separate from e-commerce with its ever-changing dynamics, researchers still believe that much more is needed to understand the perspective of new social business models.

At its inception stage, s-commerce was more concerned about the socialization considerations among consumers, whereas under new technological shifts during the past few years s-commerce is reshaping itself by drawing upon more of consumers' behavioral intents. S-commerce carries various attributes - some are related to its community building, while few drive its efficiency in the perspective of its technical manifestation. While accentuating s-commerce attributes, the extant literature has provided various insights (Yahia, Al-Neama, \& Kerbache, 2018), but leveraging on consumers' social buying intents with the synchronization of these attributes to engage them is still relatively obscure for modern-day retailers/organizations. To address the complex nature of synchronizing s-commerce attributes for achieving synergies amid consumers' thoughts, it is pertinent to discourse the parallel run of s-commerce attributes and further elucidate the in-depth appreciation of their reactions over social business models (Shen, Li, Sun, Chen, \& Wang, 2018).

S-commerce in the current era presents an opportunity for a multitude of businesses and is forecasted to continually grow into a US $\$ 80$ billion market worldwide by 2020 (Chen \& Shen, 2015). To strengthen the use of social media in e-commerce, firms such as Amazon, Alibaba, eBay, Trip.com, Qunar.com, and many others employ a strategy 
of both developing online communities and plugging-in social technologies into their websites. While incorporating new social, technological features, these websites have emerged as social and commerce platforms, thus paving a way to the concept of scommerce (Kim \& Kim, 2018). Emphasizing the adaptability of social media, past researchers believe that the principal focus of social platforms is to develop the shift of ecommerce to s-commerce and to identify the intervening constructs that resonate consumers' behavioral intents (Chen \& Shen, 2015; Hajli, 2014; Yu, Tsai, Wang, Lai, \& Tajvidi, 2018).

Certain intervening parameters exist that are discussed in the extant literature, such as satisfaction, usefulness, loyalty, trust, and much more (Bhattacherjee, 2001; Busalim \& Hussin, 2016; Chakraborty, Lee, Bagchi-Sen, Upadhyaya, \& Raghav Rao, 2016; Chang, Liu, \& Shen, 2017). Past research has tested different dimensions of these intervening constructs, but recent studies' insights argue that trust remains a challenging parameter for e-vendors when addressing the perplexing challenges of the s-commerce sphere (Connelly, Crook, Combs, Ketchen, \& Aguinis, 2018; Sharma, Menard, \& Mutchler, 2017; Yahia et al., 2018). Based on the advancements and challenges of the s-commerce era, practitioners also have the view that there must be a bifurcation in s-commerce's attributes such as community and platform. Both attributes have their own specific role in enticing consumers towards s-commerce intentions (Liang \& Turban, 2011; Zhang, Lu, Gupta, \& Zhao, 2014). In an effort to conceptualize and operationalize s-commerce attributes, the extant literature offers a broad spectrum of insights (Hajli, 2013; Zhang et al., 2014) and also presents the prospective of intervening constructs that resonate the social buying intents of consumers (Chen \& Shen, 2015; Hajli, Lin, Featherman, \& Wang, 2014; Tajvidi, Wang, Hajli, \& Love, 2017).

Community attributes, which represent a subjective psychosomatic process, describe the personal relevance and the importance of a relationship with an online community (Kim, Chan, \& Kankanhalli, 2012; Xu, Jones, \& Shao, 2009). The identification of such attributes thus facilitates the formation of the trusting mechanism towards a community that subsequently sparks a desire for s-commerce (Shen, Li, Sun, Chen, \& Wang, 2018). The most commonly adopted community attributes are forums and communities, ratings and reviews, and recommendations and referrals (Chen, Lu, \& Wang, 2017; Hajli, 2015). Apart from the s-commerce community attributes, the technological platform attributes also represent an opportunity to foster s-commerce intents (Baethge, Klier, \& Klier, 2016; 
Busalim \& Hussin, 2016; Huang \& Benyoucef, 2013) and are expected to affect scommerce behavior through the building up of trusting relationships (Liang \& Turban, 2011). Nowadays, the platform or website design derives behavioral intents among consumers (Thapliyal, 2018). Academicians have empirically presented their thoughtful arguments on the conceptualization of platform attributes (Shen et al., 2018; Zhang \& Benyoucef, 2016), but the influence of platform attributes in enticing s-commerce behavioral intents along with community attributes is still in its infancy in regards to academic insights. At stages where community and platform attributes create active and passive participation of consumers, both attributes must be judged for their roles in translating behavioral intents of consumers. Platform attributes carry more technical manifestations and may not create a higher level of trusting mechanism, thus providing more passive insightful results to practitioners in measuring consumers' intentions. Henceforth, such an understanding of s-commerce attributes creates an impetus to investigate the motives behind consumers' s-commerce behavioral intents.

Our contribution to the s-commerce literature is fourfold. First, this research underpins the role of consumers' social interactions through s-commerce community attributes (SCCA), i.e. Ratings and Reviews (RR); Recommendations and Referrals (ReRf); Forums and Communities (FC), in order to establish trust in social platform (TIP) and trust in community (TIC). Second, this study investigates the effect of the s-commerce platform attributes (SCPA), i.e. perceived interactivity (PI), perceived personalization (PP), and perceived sociability (PS), on TIP and TIC. To be more specific, we focus on three social platform attributes, extracted from extant literature, namely, perceived interactivity, perceived personalization, and perceived sociability (Zhang et al., 2014). Third, we adopt the trust transfer theory (Stewart, 2003) for treating trust as mediation towards s-commerce intention (SCI) and electronic word-of-mouth intention (e-WOMI). Since trust is an integral and exciting matter on social platforms among consumers (Hajli, Sims, Zadeh, \& Richard, 2017; Yahia et al., 2018), we believe that it should be carefully braced in the perspective of s-commerce. Fourth, building upon a systematic review of scommerce perspectives and the trust transfer theory, we propose a comprehensive framework that explains the indirect effects of s-commerce attributes on consumers' behavioral outcomes through trust. In sum, this study addresses the issues mentioned above by investigating the following research questions regarding s-commerce: 
RQ1. Do s-commerce community attributes and s-commerce platform attributes significantly influence s-commerce intention and e-WOM intention?

RQ2. How does trust (trust in social community and trust in social platform) mediate the relationship of s-commerce community attributes and s-commerce platform attributes with s-commerce intention and e-WOM intention?

The rest of the paper runs as follows: Section 2 presents the theoretical background and hypotheses development along with the representation of the theoretical framework. Section 3 discusses the research methodology, followed by the results in Section 4 . Section 5 offers a discussion and final conclusions.

\section{Theoretical Background}

S-commerce is still a new trend and subsection of e-commerce (Chen \& Shen, 2015; Hajli, Sims et al., 2017; Lin, Li, \& Wang, 2017) and permits consumers to create content over the web and enable transactions via a social media environment. As the dynamics of the Internet age are evolving at a rapid pace, researchers are trying to extract more convincing findings from the perspective of s-commerce. Blasco-Arcas (2013) presents findings of interactive platform technologies that have changed not only the structure of e-business, but also argued for how firms and customers interrelate in the marketplace with the social media plugins. Similarly, Shen et al. (2017), Hajli et al. (2017), and Zhang et al. (2014) offer findings on technological attractiveness, stimuli, and social enablers at enticing s-commerce intentions through trusting, virtual, and community involvement mechanisms. Chen et al. (2017) argue that learning from RR, ReRf, and FC resonates the cognitive and affective mechanism among s-commerce participants, which helps them in their behavioral decision making such as purchase intentions. The selected community and platform attributes have different relationships with consumers' behavioral intentions and provide varied insights through intervening constructs (Chen \& Shen, 2015; Hajli, Wang et al., 2017).

The literature has discussed many community attributes in the perspective of scommerce such as satisfaction, commitment, community services, sociability, etc. (N. Hajli et al., 2015; Kang \& Johnson, 2015), ut the most commonly adopted ones are RR, FC, and ReRf from the perspective of the Internet era's businesses (Chen et al., 2017). First, through RR it is quite convenient for consumers to give valuable reviews about the products and services they use (Ye, Law, Gu, \& Chen, 2011). Compared to e-vendor's ratings and reviews, third-party reviews are considered trustworthy (Gavilan, Avello, \& 
Martinez-Navarro, 2018). Moreover, ratings promote the phenomenon of trust, which is an integral part of our research into the shape of TIP and TIC. Second, ReRf helps at creating SCI in an online medium. Usually consumers do not experience the products or services offered by e-vendors until they read about past consumers' ReRf, thus relying on other consumers' ReRf (Hajli, 2013; Zhang, Lu, Gupta, \& Zhao, 2014). Third, for FC the discussion revolves around online communities and forums. Members of online communities browse around different groups and communities to get useful advice and feedback from existing consumers, which then lead them toward their commerce intentions (Hsu et al., 2018).

Similar to community attributes, platform attributes also shape consumers' behavioral intentions through their technical manifestation (Chen \& Shen, 2015; Yu et al., 2018). First, the interactivity of a technological medium is the degree to which one can control the medium by altering its contents in a real-time situation and create a communication medium (Hu et al., 2016; Tajvidi et al., 2017). Interactivity hence results in shaping customers' responses to an online medium with its catchy design features (Tajvidi et al., 2017). Second, personalization correlates to customers' adaptability to a website in order to cater to their preferences (Huang \& Benyoucef, 2013, 2017). In the domain of s-commerce, website personalization tactics mainly emphasize on providing online customers with tailor-made contents based on their needs, preferences, profiles, prior interactions, and social networks. Hence, it can be said that PP depicts the fit among website content and customers' preferences (Zhang et al., 2014). Third, sociability, as another technological attribute, can be explained in the following two dimensions. 1) It can be taken as the structural part of the platform attributes that creates customers' intention at engaging interdependent interactions (Chen \& Shen, 2015; Huang \& Benyoucef, 2017). 2) It can also be taken as the dynamic quality created by the communication among customers. In our approach, it is customers' perceptions in scommerce that benefits their interactions with other customers.

With their related properties, both SCCA and SCPA induce a trusting mechanism towards consumers that is considered as an integral element for structuring a successful relationship (Yahia et al., 2018). Many studies have discussed in great detail the online purchase intentions of customers through trust (Chen \& Shen, 2015; Hajli, Sims, et al., 2017; Shanmugam, Sun, Amidi, Khani, \& Khani, 2016). The trust transfer theory (Chen \& Shen, 2015; Stewart, 2003) discusses two reasons that could be put into consideration 
for creating TIP and TIC. First, trust among the community creates an understanding for believing each other through the transfer of information. Second, the existence of interpersonal trust creates trusting considerations towards an organization/business (Yahia et al., 2018). In our study, this phenomenon is very suitable as we have the intuition that both TIP and TIC are developed through SCCA and SCPA. SCPA tend to create the perception among consumers that they are being cared for by their interactivity, personalization, and sociability preferences (Kang \& Johnson, 2015). Upon developing such a perception, consumers tend to develop TIP. For TIC, it is believed that people's familiarity tends to increase upon participating in FC and reading reviews of other customers over an online medium. The extant literature argues that RR and ReRf influence the level of trust, which adds up to sales on web platforms (Li, 2017; Shanmugam et al., 2016; Sheikh, Islam, Rana, Hameed, \& Saeed, 2017). Moreover, ratings also increase the satisfaction level of consumers when they make a transaction (Gavilan et al., 2018). The present literature emphasizes that trust towards community members positively affects the participating behavior of customers, such as information sharing in social FC (Ridings, Gefen, \& Arinze, 2002; Shen, Lee, \& Cheung, 2014).

When community and platform attributes positively impact consumers' behavioral intentions through a trusting mechanism, retailers/organizations gain commercial benefits, which may be measured by an increase in transactions or customer loyalty. However, we believe that SCI is derived from consumers' benefit or trusting beliefs that get created during online transactions (Akram, Malik, Akhter, \& Shakir, 2019; Shareef, Kumar, Kumar, \& Dwivedi, 2011; Zhu et al., 2018). Similarly, e-WOMI is considered in online mediums, communities, and forums. The extant literature (Lin et al., 2017; Ortiz, Chih, \& Teng, 2017) has coined the term online WOM intention, which is defined as customers' experiences and opinions shared with online members under the use of online technologies. Compared to WOM, e-WOM is fast and convenient and can create help for many customers.

\subsection{Development of hypotheses and the conceptual model}

This section presents logical argumentations to support the hypothetical intuition sets that are drafted among selected latent constructs - i.e., s-commerce community attributes, s-commerce platform attributes, trust in community, and trust in platform, s-commerce intention, and e-WOMI. 


\subsubsection{S-commerce community attributes (SCCA) and s-commerce intention (SCI)}

SCCA enable communication among customers and create a mechanism for customer relationship management (CRM) (Huang \& Benyoucef, 2013; Ng, 2013). Once the communication among customers gets started, they will interact more socially with each other. This social interaction creates an opportunity for customers to gain more knowledge and create trust (Lin, Yan, Chen, \& Luo, 2017). Members of a social community can reassure each other through the information exchange, thus increasing their trust and purchase intention (Chen, Su, \& Widjaja, 2016; Kim \& Kim, 2018). Past studies argue that social frameworks and social support do influence trust (Hajli, 2014; Yahia et al., 2018). Moreover, customers show their inclination towards trust when they get informational and emotional support (Li \& Ku, 2018; Lin et al., 2017) and also amplify their buying intentions. When people participate in FC or read others' RR for a product or service, their level of familiarity to a website or social platform is likely to increase, thus prompting SCI. Past studies also advocate that there exists a significant relationship between trust and online commerce behavior (Ng, 2013; Shanmugam et al., 2016). Hence, based on the above literature insights, we hypothesize the following.

H1: Trust (in platforms and in community) mediates the relationships between $s$ commerce community attributes (SCCA) (a: ratings and reviews (RR), b: recommendations and referrals (ReRf), $c$ : forums and communities (FC)) and s-commerce intentions (SCI).

\subsubsection{S-commerce community attributes (SCA) and e-WOM intention (e-WOMI)}

Social sites provide customers with many ways to communicate with each other for gaining trust (Shanmugam et al., 2016). They create successful relationships through active communication and also have a positive effect on trust (Li, 2017; Ortiz et al., 2017). Customers who share their experiences and information on social platforms in the form of recommendations and referrals are more likely to create trust over online shopping platforms (Chen \& Shen, 2015; Kim \& Park, 2013). While reading past customers' ReRf, potential customers value the shared information, thus making an appropriate purchase decision (Shanmugam et al., 2016). The extant literature demonstrates that consumers on FC with high trusting factors also bear the propensity to spread positive e-WOMI (Kim \& Park, 2013). As potential consumers usually shop for products and services under the influence of past customers' suggestions and opinions, upon experiencing similar experiences they become prone to share WOM (Choi, Seo, \& Yoon, 2017). Thus, customers with increased trust in an s-commerce website are more likely to share their experiences 
with existing and prospective customers through the online platform. For this reason, we also believe that trust could influence e-WOMI as well. Hence, based on the above literature insights, we hypothesize the following.

H2: Trust (in platforms and in community) mediates the relationship between $s$ commerce community attributes (SCCI) (a: ratings and reviews (RR), b: recommendations and referrals (ReRf), c: forums and communities (FC)) and e-WOM intentions (e-WOMI).

\subsubsection{S-commerce platform attributes SCPA) and s-commerce intention (SCI)}

With the addition of technological plugins over s-commerce platforms, customers are more keen at enhancing their interactivity, personalization, and sociability in an scommerce environment (Lin et al., 2017). Perceived interactivity, personalization, and sociability create stimuli for consumers for attaining social support and trust, thus forming s-commerce intentions (Zhang et al., 2014). In attaining social support through the enabled technological frameworks on websites, customers carry the susceptibility of creating trust about platform (Chen \& Shen, 2015). Social support not only creates trust, but it also leads to SCI and e-WOMI (Chen \& Shen, 2015). With the explained objective and subjective aims in the extant literature, collectively SCPA create the necessary social care for customers and enhance TIC and TIP (Dholakia \& Talukdar, 2004). From the gained trust through SCPA, customers also attain the power of control over their transaction (Farivar, Turel, \& Yuan, 2017). This power supports in shaping customers' interaction with social platforms, thus increasing their SCI. Hence, based on the above literature insights, we hypothesize the following.

H3: Trust (in platforms and in community) mediates the relationship between $s$ commerce platform attributes (SCPA) (a: perceived interactivity, b: perceived sociability, c: perceived personalization) and s-commerce intentions (SCI).

\subsubsection{S-commerce platform attributes (SCPA) and e-WOM intention (eWOMI)}

Platform attributes do create e-WOMI through TIP and TIC (Zhang et al., 2014) and provide a mechanism to customers, which upon browsing social network site create social support for them (Chen \& Shen, 2015; Chen et al., 2016; Yahia et al., 2018). Social support not only creates trust, but also leads to s-commerce sharing intentions (Shanmugam et al., 2016; Zhang et al., 2014). While we have discussed earlier that all SCPA have their own specific role in creating trust, they also create social care for customers. With the reception of social care, customers reciprocate the care in terms of e-WOMI. Therefore, customers with a high level of TIP or TIC are likely to share their 
experiences in the form of e-WOMI (Kim \& Park, 2013). Hence, based on the above discussions, we hypothesize the following.

H4: Trust (in platforms and in community) mediates the relationship between $s$ commerce platform attributes (SCPA) (a: perceived interactivity, b: perceived sociability, $c$ : perceived personalization) and e-WOM intentions (e-WOMI).

Previous literature also suggests that gender, age, income, and experience may affect purchase intention on the Internet (Gibreel, AlOtaibi, \& Altmann, 2018; Zhang et al., 2014). The present study thus includes gender, age, income, and s-commerce experience as control variables in the model. Furthermore, Figure 1 illustrates the research model that represents the intuition set used herein.

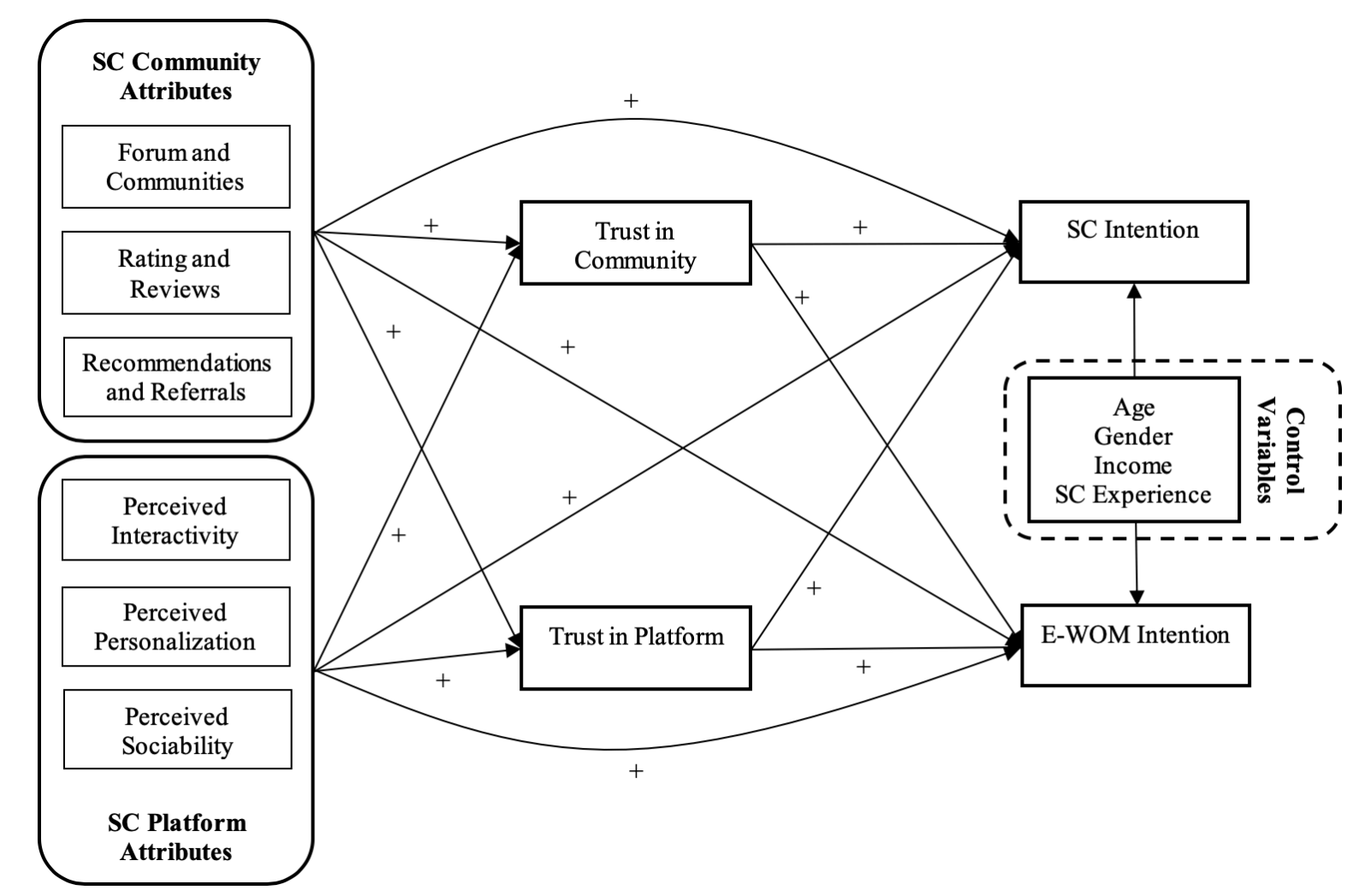

Note: $S C=$ Social commerce, $E-$ WOM= Electronic word of mouth

Figure 1 Theoretical framework

\section{Methodology}

This methodology section specifies the data collection mechanism and the descriptive statistics of the respondents.

\subsection{Data collection}

We adapt multi-item scales from prior and similar s-commerce research to measure each of the latent variables. While the questionnaire was originally developed in English, it was 
then translated into Chinese to help the respondents better understand it. We follow the translation and back-translation approach to ensure the linguistic equivalence of the instruments used. Several faculty members and doctoral students reviewed the initial version of the questionnaire and provided feedback on content and clarity of instructions. Their feedback led to several changes in the item wording and the final version. In order to check the face validity of survey instruments, we refined the questionnaire wording, assessed logical consistencies, judged ease of understanding, and identified areas for improvement. Overall, we regard the questionnaire as concise and easy to complete. We employ a 7-point Likert scale on all items (except the demographics) and list the measurement items used in Appendix A.

We utilize data for holiday booking websites (Qunar, Trip, and others) from an online survey via Sojump [http://www.sojump.com], which is a popular online survey platform in China. After presenting potential respondents with the definitions of SCCA, SCPA, TIP, and TIC in the survey questionnaire, we inserted a filtering question inquiring about the respondents' s-commerce experience: "How long have you been using s-commerce to book holidays/ticketing?" We only permitted respondents with prior experience at scommerce use on holiday booking websites to continue with the survey. The process yielded a total of 822 responses. To ensure that only one response was submitted per respondent, each participant's Internet protocol (IP) address and demographic information were recorded and carefully examined. A pilot test of 20 respondents suggested that at least five minutes were needed to complete the questionnaire. Hence, 21 questionnaires completed in less than five minutes were abandoned, resulting in 801 responses. After eliminating 17 outliers based on Mahalanobis distance, we take the final 784 valid responses for further analysis. Through the statistics provided by sojump.com, we find that 252 participants answered our questionnaire employing a personal computer and the other 532 answers did so from mobile phones/devices.

\subsection{Data characteristics}

Table 1 depicts the following sample characteristics: $42.1 \%$ of the respondents are male and $57.9 \%$ are female; $36.6 \%$ are under 20 years old, $38.8 \%$ are between 21 to 30 years old, while $24.6 \%$ are above 30 years old. In the education statistics, $16.1 \%$ respondents have a high school education, $40.3 \%$ have a college education, and $43.6 \%$ have a university education. For employment, $25.4 \%$ are students, $22.4 \%$ are public employees, $21.8 \%$ are private sector employees, $18.4 \%$ are self-employed, and $12 \%$ are others. For 
income, $34.1 \%$ of the respondents earn less than $4000 \mathrm{RMB}, 28.4 \%$ earn between 4001 and $6000 \mathrm{RMB}, 26.7 \%$ earn between 6001 and $8000 \mathrm{RMB}$, and 10.8\% earn over 8000 RMB per month. For s-commerce experience, 19.8\% of respondents have under 1 year of experience, 42.1\% have between 1 and 3 years, 21.2\% have between 3 and 5 years, and $17 \%$ have over 5 years. These data-points testify that most survey respondents have an acceptable knowledge of s-commerce. Moreover, s-commerce usage per month was also taken, with $27.8 \%, 36.7 \%$, and $35.5 \%$ of respondents conducting it less than 2 times, 3 to 4 times, and 5 times or more, respectively. Lastly, website preferences for booking holidays are $43.9 \%, 39 \%$, and $17.1 \%$ for Qunar, Trip, and others.

Table 1 Sample characteristics

\begin{tabular}{|c|c|c|c|}
\hline Measure & Item & Frequency & Percentage \\
\hline \multirow[t]{2}{*}{ Gender } & Male & 330 & 42.1 \\
\hline & Female & 454 & 57.9 \\
\hline \multirow[t]{3}{*}{ Age } & Less than 20 Years old & 287 & 36.6 \\
\hline & 21 to 30 years old & 304 & 38.8 \\
\hline & More than 30 years old & 193 & 24.6 \\
\hline \multirow[t]{3}{*}{ Education } & High school & 126 & 16.1 \\
\hline & College & 316 & 40.3 \\
\hline & University & 342 & 43.6 \\
\hline \multirow[t]{5}{*}{ Employment } & Student & 199 & 25.4 \\
\hline & Public employee & 176 & 22.4 \\
\hline & Private sector employee & 171 & 21.8 \\
\hline & Self-employed & 144 & 18.4 \\
\hline & Others & 94 & 12.0 \\
\hline Income & Less than $4000 \mathrm{RMB}$ & 267 & 34.1 \\
\hline \multirow[t]{3}{*}{ (monthly) } & Between 4001 and 6000 RMB & 223 & 28.4 \\
\hline & Between 6001 and $8000 \mathrm{RMB}$ & 209 & 26.7 \\
\hline & Above 8000 & 85 & 10.8 \\
\hline \multirow{4}{*}{$\begin{array}{l}\text { S-commerce } \\
\text { experience }\end{array}$} & Less than 1 year & 155 & 19.8 \\
\hline & Between 1 and 3 years & 330 & 42.1 \\
\hline & Between 3 and 5 years & 166 & 21.2 \\
\hline & More than 5 years & 133 & 17.0 \\
\hline \multirow{3}{*}{$\begin{array}{l}\text { S-commerce } \\
\text { usage per month }\end{array}$} & Less than 2 times & 218 & 27.8 \\
\hline & 3 to 4 times & 288 & 36.7 \\
\hline & 5 times or more & 278 & 35.5 \\
\hline \multirow{3}{*}{$\begin{array}{l}\text { Preferred s- } \\
\text { commerce } \\
\text { platform }\end{array}$} & Qunar.com & 344 & 43.9 \\
\hline & Trip.com & 306 & 39.0 \\
\hline & Others & 134 & 17.1 \\
\hline
\end{tabular}

$N=784$. 


\section{Analysis and Results}

In view of our conceptual model, we use structural equation modeling (SEM) to analyze the direct and indirect effects. In business research, SEM has become quite prevalent due to its advantages of testing the measurement and the structural models, thus allowing the researcher to test the psychometric properties of the scale and causal relationships (Hair, Ringle, \& Sarstedt, 2011). Covariance-based structural equation modeling (CB-SEM), having no limitation with respect to goodness of model fit measures, is a preferred technique for theory testing and confirmation as compared to the less rigorous variancebased partial least squares approach (Hair et al., 2011). Thus, we opt to utilize CB-SEM for validating the measurement properties and testing the hypothesized relations. In this pursuit, conforming to the two-step for model testing by Anderson and Gerbing (1988) and before testing the proposed hypotheses - through a structural regression model psychometric properties of the latent constructs have been assessed through the CB-SEM approach.

\subsection{Psychometric properties and instrument validation}

We carry out confirmatory factor analysis, together with all the latent constructs, to test the psychometric properties of the scale. An assessment of the measurement model's goodness of fit indices indicates an adequate model fit, and that the model fit measures $\left[\chi^{2} / \mathrm{df}=1.844\right.$; goodness of fit index $(\mathrm{GFI})=0.938$; comparative fit index $(\mathrm{CFI})=0.971$; Tucker-Lewis index $(\mathrm{TLI})=0.966$; root mean square error of approximation $($ RMSEA) $=$ 0.033; standardized root mean square residual $(\mathrm{SRMR})=0.031$ ] are under the recommended levels (Hu \& Bentler, 1999). For assessing the psychometric properties of the instrument, its reliability is assessed in terms of composite reliability $(\rho)$ and internal consistency $(\alpha)$, while its validity is assessed by convergent and discriminant validities (Fornell \& Larcker, 1981; Nunnally, 1978). For scale validity, Cronbach's alpha and the composite reliability values are assessed and found to be higher than 0.7 for all the constructs, thus establishing the reliability of the scale (Table 2).

\subsection{Instrument validation}

This research examines the instrument validation by estimating the initial reliability check of each item at the construct level. At the item level, factor loadings of each item are all above the recommended value of 0.6 (Gerbing \& Anderson, 1988). At the construct level, the internal consistency (Cronbach's alpha) and composite reliability (CR) values are well above 0.70 , thus confirming the reliability of all the constructs (Nunnally, 1978). 
Additionally, significant item loadings on their designated latent variables and average variance extracted (AVE) are greater than 0.50, suggesting the convergent validity of the scale (Fornell \& Larcker, 1981; Gerbing \& Anderson, 1988). Both of these conditions are met, thus establishing convergent validity (Table 2). 
Table 2 Constructs' reliability and validity

\begin{tabular}{|c|c|c|c|c|c|}
\hline Construct & Item & $\begin{array}{c}\text { Std. } \\
\text { Loading }\end{array}$ & CA & CR & AVE \\
\hline \multirow[t]{3}{*}{ Ratings and reviews (RR) } & RR1 & 0.732 & 0.832 & 0.837 & 0.632 \\
\hline & RR2 & 0.833 & & & \\
\hline & RR3 & 0.816 & & & \\
\hline \multirow{3}{*}{$\begin{array}{l}\text { Recommendation and } \\
\text { referrals (ReRf) }\end{array}$} & ReRf1 & 0.788 & 0.842 & 0.847 & 0.648 \\
\hline & ReRf2 & 0.851 & & & \\
\hline & ReRf3 & 0.774 & & & \\
\hline \multirow{3}{*}{$\begin{array}{l}\text { Forums and communities } \\
\text { (FC) }\end{array}$} & FC1 & 0.828 & 0.823 & 0.826 & 0.613 \\
\hline & $\mathrm{FC} 2$ & 0.804 & & & \\
\hline & $\mathrm{FC} 3$ & 0.712 & & & \\
\hline \multirow[t]{3}{*}{ Perceived interactivity (PI) } & PI1 & 0.723 & 0.810 & 0.814 & 0.594 \\
\hline & PI2 & 0.826 & & & \\
\hline & PI3 & 0.758 & & & \\
\hline \multirow{4}{*}{$\begin{array}{l}\text { Perceived personalization } \\
\text { (PP) }\end{array}$} & PP1 & 0.740 & 0.831 & 0.832 & 0.555 \\
\hline & PP2 & 0.780 & & & \\
\hline & PP3 & 0.784 & & & \\
\hline & PP4 & 0.670 & & & \\
\hline \multirow{4}{*}{ Perceived sociability (PS) } & PS1 & 0.849 & 0.915 & 0.919 & 0.726 \\
\hline & PS2 & 0.863 & & & \\
\hline & PS3 & 0.840 & & & \\
\hline & PS4 & 0.855 & & & \\
\hline \multirow[t]{4}{*}{ Trust in community (TC) } & TIC1 & 0.716 & 0.814 & 0.819 & 0.531 \\
\hline & TIC2 & 0.698 & & & \\
\hline & TIC3 & 0.728 & & & \\
\hline & TIC4 & 0.771 & & & \\
\hline \multirow[t]{4}{*}{ Trust in platform (TP) } & TIP1 & 0.831 & 0.858 & 0.861 & 0.660 \\
\hline & TIP2 & 0.799 & & & \\
\hline & TIP3 & 0.794 & & & \\
\hline & TIP4 & 0.691 & & & \\
\hline \multirow[t]{3}{*}{ S-commerce intention (SCI) } & SCI1 & 0.784 & 0.783 & 0.782 & 0.547 \\
\hline & $\mathrm{SCI} 2$ & 0.714 & & & \\
\hline & SCI3 & 0.719 & & & \\
\hline \multirow{3}{*}{$\begin{array}{l}\text { e-WOM intention (E- } \\
\text { WOMI) }\end{array}$} & E-WOMI1 & 0.784 & 0.863 & 0.865 & 0.681 \\
\hline & E-WOMI2 & 0.845 & & & \\
\hline & E-WOMI3 & 0.845 & & & \\
\hline
\end{tabular}

Discriminant validity can be established when the measures of each construct converge on their respective true scores, which are uniquely distinct from those of the others. We check discriminant validity by examining factor correlations (Kline, 2010) and if the square root of the average variance extracted for each construct is larger than its correlation with the other factors (Fornell \& Larcker, 1981). All inter-construct correlations in our analysis are less than 0.6 , and the square root of each construct's 
average is significantly higher than the correlation between any pair of factors, confirming the discriminant validity of the scale (Table 3).

Table 3 Factor correlation coefficients and square roots of AVE

\begin{tabular}{|c|c|c|c|c|c|c|c|c|c|c|}
\hline Construct & RR & FC & ReRf & PP & PS & PI & TIC & TIP & SCI & E-WOMI \\
\hline $\mathbf{R R}$ & 0.795 & & & & & & & & & \\
\hline FC & $0.471^{* * *}$ & 0.783 & & & & & & & & \\
\hline ReRf & $0.381^{* * *}$ & $0.478^{* * *}$ & 0.805 & & & & & & & \\
\hline PP & $0.399 * * *$ & $0.415^{* * *}$ & $0.507^{* * *}$ & 0.745 & & & & & & \\
\hline PS & $0.264^{* * *}$ & $0.327^{* * *}$ & $0.381^{* * *}$ & $0.415^{* * *}$ & 0.852 & & & & & \\
\hline PI & $0.227^{* * *}$ & $0.376^{* * *}$ & $0.366^{* * *}$ & $0.383^{* * *}$ & $0.338^{* * *}$ & 0.770 & & & & \\
\hline TIC & $0.459^{* * *}$ & $0.551^{* * *}$ & $0.486^{* * *}$ & $0.464^{* * *}$ & $0.416^{* * *} \mathrm{C}$ & $0.470^{* * *}$ & 0.729 & & & \\
\hline TIP & $0.413^{* * *}$ & $0.442^{* * *}$ & $0.490^{* * *}$ & $0.489^{* * *}$ & $0.536^{* * *} c$ & $0.409^{* * *}$ & $0.662^{* * *}$ & 0.781 & & \\
\hline SCI & $0.456^{* * *}$ & $0.431^{* * *}$ & $0.396^{* * *}$ & $0.426^{* * *}$ & $0.414^{* * *} C$ & $0.370^{* * *}$ & $0.614^{* * *} 0$ & $0.569 * * *$ & 0.740 & \\
\hline
\end{tabular}

Notes: $\quad R R=$ Ratings and reviews, ReRf=Recommendations and referrals, FC=Forums and communities, PI=Perceived interactivity, $P P=$ Perceived personalization, $P S=$ Perceived sociability, $T I C=$ Trust in community, $T I P=$ Trust in platform, $S C I=S$-commerce intention, e$W O M I=e-W O M$ intention. The numbers in the diagonal line represent the square root of the average variance extracted, whereas the offdiagonal values are the inter-construct correlations. $* * * p<0.001$.

\subsection{Common method variance}

We next assess common method variance (CMV), because there exists a consensus that data collected at one point in time for independent and dependent variables may possess it (Podsakoff, MacKenzie, Lee, \& Podsakoff, 2003). Several procedural and statistical measures suggested by (Podsakoff et al., 2003) are used to minimize CMV. First, participants were given the assurance of secrecy and discretion of responses they provide, to limit concerns about evaluation hesitation and social desirability. Second, a psychometric separation was constructed in the survey with the aim of reducing the participants' perception of any direct connection between these constructs. This is achieved by giving different sets of instructions to the respondents. Finally, we test the potential influence of CMV statistically using Harman's one-factor test in the SPSS 23 software. We perform principal factor analysis without rotation to determine whether a single factor explains the majority of the variance. The results report more than one factor with an Eigen value of greater than one, with the first factor accounting for $31.138 \%$ of the total variance explained. Thus, CMV does not appear to be a serious problem in this study. 


\subsection{Testing of the structural model and hypotheses}

Having established the reliability and validity of the measurement model, we continue to assess the structural model and employ a maximum likelihood estimate through AMOS 24. The assessment findings on the structural model reveal an adequate fit, as the values of various fit indices are within the range for an acceptable model fit $\left[\chi^{2} / \mathrm{df}=1.842\right.$, GFI $=0.930, \mathrm{AGFI}=0.915, \mathrm{CFI}=0.963, \mathrm{TLI}=0.957, \mathrm{RMSEA}=0.033$, and Standardized $\mathrm{RMR}=$ 0.039]. Moreover, $\mathrm{R}^{2}$ values for TIP, TIC, SCI, and e-WOMI are 47.6\%, 49.2\%, 50.2\%, and $47.2 \%$, respectively.

\subsection{Direct and indirect effects}

To assess the mediating effects of trust in platforms and in community on the relationship between s-commerce drivers and behavioral outcomes, we examine the direct and indirect effects of SCCA and SCPA on SCI and e-WOMI through TIC and TIP. We use the bootstrap procedure with maximum likelihood to calculate bias-corrected confidence intervals. Table 4 summarizes the direct effects of various relationships in the conceptual model.

Table 4 Direct effects

\begin{tabular}{|c|c|c|c|c|c|c|c|c|}
\hline \multirow[t]{2}{*}{ Predictor } & \multicolumn{2}{|c|}{ TIP } & \multicolumn{2}{|c|}{ TIC } & \multicolumn{2}{|c|}{ SCI } & \multicolumn{2}{|c|}{ e-WOMI } \\
\hline & Estimate & T-Value & Estimate & T-Value & Estimate & T-Value & Estimate & T-Value \\
\hline FC & $0.104+$ & 1.926 & $0.247^{* * *}$ & 4.333 & $0.009 \mathrm{~ns}$ & 0.173 & $-0.047 \mathrm{~ns}$ & -0.959 \\
\hline ReRf & $0.157^{* *}$ & 3.078 & $0.132^{* *}$ & 2.750 & $-0.019 \mathrm{~ns}$ & -0.328 & $-0.036 n s$ & -0.750 \\
\hline $\mathrm{RR}$ & $0.148^{* * *}$ & 3.364 & $0.179^{* *}$ & 3.086 & $0.170^{* * *}$ & 3.696 & $0.246^{* * *}$ & 5.348 \\
\hline PP & $0.133^{* *}$ & 2.608 & $0.093+$ & 1.632 & $0.03 \mathrm{~ns}$ & 0.526 & $0.008 \mathrm{~ns}$ & 0.195 \\
\hline PS & $0.304^{* * *}$ & 7.070 & $0.135^{* *}$ & 3.140 & $0.091+$ & 1.750 & $0.101^{*}$ & 2.295 \\
\hline PI & $0.138^{* *}$ & 3.000 & $0.217^{* * *}$ & 4.717 & $0.046 \mathrm{~ns}$ & 0.836 & $0.217^{* * *}$ & 4.717 \\
\hline TIC & & & & & $0.342^{* * *}$ & 5.344 & $0.218^{* * *}$ & 3.964 \\
\hline TIP & & & & & $0.215^{* *}$ & 3.359 & $0.237^{* * *}$ & 4.472 \\
\hline Age & & & & & $0.031 \mathrm{~ns}$ & 0.912 & $-0.006 \mathrm{~ns}$ & -0.214 \\
\hline Gender & & & & & $0.058+$ & 1.758 & $0.002 \mathrm{~ns}$ & 0.069 \\
\hline Income & & & & & $0.035 \mathrm{~ns}$ & 1.000 & $0.020 \mathrm{~ns}$ & 0.714 \\
\hline SCE & & & & & $0.094^{* *}$ & 2.765 & $0.104^{*}$ & 3.586 \\
\hline
\end{tabular}

Notes: RR=Ratings and reviews, ReRf=Recommendations and referrals, FC=Forums and communities, PI=Perceived interactivity, $P P=$ Perceived personalization, $P S=$ Perceived sociability, TIC=Trust in community, TIP=Trust in platform, $S C I=S$-commerce intention, $e$ $W O M I=e-W O M$ intention, $S C E=S$-commerce experience.

Significance of Correlations: ${ }^{* * *} p<0.001,{ }^{* *} p<0.010,{ }^{*} p<0.050$, and $t p<0.100$.

Table 5 summarizes the indirect effects of SCCA and SCPA on SCI and e-WOMI through TIC and TIP. 
Table 5 Indirect effects

\begin{tabular}{|c|c|c|c|c|c|c|c|c|c|}
\hline \multirow{2}{*}{ Predictor } & \multirow{2}{*}{ Mediator } & \multicolumn{4}{|c|}{ SCI } & \multicolumn{4}{|c|}{ e-WOMI } \\
\hline & & Estimate & BCCIL & BCCIU & $\mathrm{P}$ & Estimate & BCCIL & BCCIU & $\mathrm{P}$ \\
\hline $\mathrm{RR}$ & TIC & 0.054 & 0.023 & 0.095 & 0.007 & 0.033 & 0.015 & 0.060 & 0.007 \\
\hline $\mathrm{RR}$ & TIP & 0.028 & 0.010 & 0.048 & 0.012 & 0.030 & 0.013 & 0.050 & 0.009 \\
\hline ReRf & TIC & 0.047 & 0.017 & 0.083 & 0.011 & 0.029 & 0.012 & 0.057 & 0.007 \\
\hline ReRf & TIP & 0.035 & 0.015 & 0.072 & 0.003 & 0.037 & 0.015 & 0.065 & 0.005 \\
\hline FC & TIC & 0.095 & 0.053 & 0.141 & 0.012 & 0.058 & 0.026 & 0.096 & 0.014 \\
\hline FC & TIP & 0.025 & 0.004 & 0.060 & 0.031 & 0.026 & 0.003 & 0.051 & 0.054 \\
\hline PP & TIC & 0.037 & 0.006 & 0.091 & 0.054 & 0.023 & 0.004 & 0.055 & 0.054 \\
\hline PP & TIP & 0.034 & 0.011 & 0.066 & 0.007 & 0.036 & 0.012 & 0.064 & 0.006 \\
\hline PS & TIC & 0.043 & 0.016 & 0.073 & 0.018 & 0.026 & 0.010 & 0.052 & 0.010 \\
\hline PS & TIP & 0.061 & 0.031 & 0.100 & 0.013 & 0.065 & 0.042 & 0.105 & 0.007 \\
\hline PI & TIC & 0.080 & 0.051 & 0.124 & 0.004 & 0.049 & 0.031 & 0.084 & 0.002 \\
\hline PI & TIP & 0.032 & 0.012 & 0.063 & 0.005 & 0.034 & 0.016 & 0.067 & 0.003 \\
\hline
\end{tabular}

Notes: RR=Ratings and reviews, ReRf=Recommendations and referrals, $F C=$ Forums and communities, PI=Perceived interactivity, $P P=$ Perceived personalization, $P S=P e r c e i v e d$ sociability, $T I C=$ Trust in community, $T I P=$ Trust in platform, $S C I=S$-commerce intention, $e$ WOMI=e-WOM intention, BCCIL/U= Bias Corrected Confidence Interval Lower/Upper.

In H1, we propose that TIC and TIP mediate the relationship between SCCA and SCI. To verify this, we check the direct and indirect effects of SCCA on SCI through TIP and TIC. All indirect effects of s-commerce community attributes though TIC $[R R(\beta=.054, P<.01)$, $\operatorname{ReRf}(\beta=.047, \mathrm{P}<.05)$ and FC $(\beta=.095, \mathrm{P}<.05)]$ and TIP $[\operatorname{RR}(\beta=.028, \mathrm{P}<.05), \operatorname{ReRf}(\beta$ $=.035, \mathrm{P}<.01)$ and FC $(\beta=.025, \mathrm{P}<.05)]$ on SCI are positive and significant. This indicates that TIC and TIP mediate the relationship between SCCA and SCI. Moreover, the direct effect of RR $(\beta=.170, P<.001)$ on $S C I$ is significant, while the direct effects of ReRf and FC on SCI are insignificant. Therefore, we conclude full mediation for FC and ReRf, whereas partial mediation exists for RR. Overall, the results support the mediating effects of TIC and TIP on the relationship between SCA and SCI, thereby supporting H1.

In $\mathrm{H} 2$, we propose that TIC and TIP mediate the relationship between SCCA and eWOMI. To confirm this, we adopt a similar procedure as for H1's analysis, i.e. the direct and indirect effects between SCCA and e-WOMI. The indirect effects of SCCA through TIC $[\operatorname{RR}(\beta=.033, \mathrm{P}<.01), \operatorname{ReRf}(\beta=.029, \mathrm{P}<.01)$, and $\mathrm{FC}(\beta=.058, \mathrm{P}<.05)]$ as well as through $\operatorname{TIP}[\operatorname{RR}(\beta=.030, \mathrm{P}<.01), \operatorname{ReRf}(\beta=.037, \mathrm{P}<.01)$, and FC $(\beta=.026, \mathrm{P}<.10)]$ are all positive and significant. In addition, the direct effects of SCCA on e-WOMI are also checked to verify partial or full mediation. The direct effect of $\operatorname{RR}(\beta=.246, \mathrm{P}<.001)$ is significant, but the direct effects of ReRf and FC are not. Thus, TIP and TIC fully mediate the relationship between ReRf and e-WOMI as well as between FC and e-WOMI, but partially mediate the relationship between RR and e-WOMI, therefore supporting $\mathrm{H} 2$. The 
results are consistent with the extant literature, which suggests that trust is an integral part of online business and could be treated as a mediating variable (Stewart, 2003). These results also suggest that SCI and e-WOMI increase upon the inclusion of trust as a mediating variable (Hajli et al., 2014). Our analysis investigates that trust created by community attributes not only increases SCI, but also spurs the intention of consumers to spread the word around.

In the case of SCPA, two similar hypotheses are drafted. In H3, we propose that TIC and TIP mediate the relationship between SCPA and SCI. To confirm that, we assess the indirect effects of SCPA on SCA though TIC and TIP. The results confirm the significant indirect effects of SCPA on SCA through TIC [PP $(\beta=.037, \mathrm{P}<.10)$, PS $(\beta=.043, \mathrm{P}<.05)$, PI $(\beta=.080, P<.01)]$ as well as through TIP [PP $(\beta=.034, \mathrm{P}<.01)$, PS $(\beta=.061, \mathrm{P}<.05)$, PI $(\beta=.032, P<.01)]$. Further results indicate no significant direct effects of any SCPA on SCI except PS $(\beta=.091, \mathrm{P}<.10)$. Thus, TIC and TIP partially mediate the relationship between PS and SCI, while full mediation is established on the relationship between PP and SCI as well as between PI and SCI, thereby supporting H3.

In $\mathrm{H} 4$, we propose that TIC and TIP mediate the relationship between SCPA and eWOMI. The indirect effects of SCPA on e-WOMI through TIC [PP $(\beta=.023, \mathrm{P}<.10)$, PS ( $\beta$ $=.026, \mathrm{P}<.05), \mathrm{PI}(\beta=.049, \mathrm{P}<.01)]$ as well as through $\mathrm{TIP}[\mathrm{PP}(\beta=.036, \mathrm{P}<.01), \mathrm{PS}(\beta$ $=.065, \mathrm{P}<.05), \mathrm{PI}(\beta=.034, \mathrm{P}<.01)]$ are significant. Apart from $\mathrm{PP}$, the direct effects for PS $(\beta=.101, \mathrm{P}<.05)$ and PI $(\beta=.217, \mathrm{P}<.001)$ are also significant. This confirms the partial mediation effects of TIC and TIP on the relationship between PS and e-WOMI as well as between PI and e-WOMI, while there are full mediation effects of TIC and TIP on the relationship between PP and e-WOMI, hence supporting H4. Concerning the control variables, we do not find any effects of gender, age, or income on SCI or e-WOMI. However, users' social commerce has significant positive effects on SCI $(\beta=.094, \mathrm{P}<.01)$ and eWOMI $(\beta=.104, \mathrm{P}<.05)$.

The above results suggest that TIC and TIP play a significant role in both SCI and eWOMI. Trust is an integral and exciting aspect of social platforms and can be used in the s-commerce mechanism, because social platforms create interactions among consumers, topping up the level of trust (Hajli, 2014; Hajli, 2015). This suggests that trust is an integral facet of any online business medium and does positively impact SCI and e-WOMI. 


\section{Discussion and Conclusion}

The primary focus of this paper is to investigate the roles of SCCA and SCPA in SCI and eWOMI and to examine the mediating effects of TIC and TIP on the relationships between s-commerce attributes (community/platform) and behavioral outcomes (SCI and eWOMI). This study contributes to the s-commerce literature by empirically establishing the significant intervening roles of TIC and TIP in developing consumers' positive SCI and e-WOMI. The results herein are consistent with past studies (Chen, Su, \& Widjaja, 2016; Kim \& Kim, 2018; Li \& Ku, 2018; Lin et al., 2017; Li, 2017; Ortiz et al., 2017) that SCCA and SCPA have indirect impacts on SCI and e-WOMI (Table 5). However, FC, ReRf, and PP have insignificant direct effects on SCI and e-WOMI, whereas PI has an insignificant direct effect on only SCI. A plausible reason for the insignificant direct effects could be that FC and ReRf are constructs that are closely related to the social consciousness of consumers when buying or utilizing products or services, and hence said constructs impact SCI and e-WOMI through some intervening factors such as trust in our study. Moreover, these insignificant direct relationships of said constructs also advocate the intuition that RR and ReRf are not traditional e-commerce constructs; instead, they carry more social manifestation and are inherently social-oriented. Thus, SCI and e-WOMI can be embraced only through some intervening constructs. PP and PI also carry social manifestation in addition to their technical nature, and PP and PI are platform attributes, which usually consumers use to explore people with shared interests, backgrounds, and similar shopping habits. Such social and technical manifestations of PP and PI create informal and socio-emotional communication among people within the community and impact SCI and e-WOMI through a trust mechanism.

Our results generally confirm all of our hypotheses. Built upon the trust transfer theory and previous studies related to s-commerce, the present research also confirms the mediating role of trust in the relationship between both online community and platform attributes and both SCI and e-WOMI. This provides us with a rationale that the synchronization of both SCCA and SCPA in new business models helps provide more insightful results to practitioners. Our study's outcomes also points out that the technical side of s-commerce business models must be treated carefully so as to translate into more convincing consumer behavioral intents.

The path coefficients suggest that SCPA perform in different aspects. First, PI helps at getting control of the online medium by allowing consumers to shape its contents in a 
real-time situation. Second, PP increases consumers' adaptability of the website, helps cater to their preferences, and achieves the right fit among website content and their preferences, which is consistent with the findings of Zhang et al. (2014). Finally, PS facilitates the social sharing among customers. From our analysis we also believe that the discussed platform attributes also decrease behavioral hesitation among customers by enabling different technological tools such as web 2.0 and social media on websites. These tools both stimulate trust among members and create intentions to buy and share information (Um, 2018). SCCA and SCPA together can make a desirable relationship of business modeling, as together they provide trust for consumers. Therefore, their combination may evoke interest and curiosity among customers who seek useful information under buying and sharing intentions. The synchronization of both attributes creates ease-of-use and conclusively acts like social support to customers in the online medium, thus forming a high level of trust. Therefore, consumers with a high level of trust in SCCA and SCPA are likely to pursue their buying intentions appropriately and share their experiences.

\subsection{Theoretical and managerial implications}

This study contributes to the current understanding of s-commerce research in several ways. Regarding theoretical implications, this research proposes a new model in the scommerce domain by exploring SCCA and SCPA as predictors of SCI and e-WOMI. Moreover, using the trust transfer theory, the study highlights the role of consumers' trust in this relationship. First, the study extends the literature of SCCA, SCPA, and trust bifurcation. Despite extant literature already available on SC, tp the best of our knowledge, the comparative effects of SCCA and SCPA on SCI and e-WOMI through TIC and TIP have never been tested before. Second, little research has been conducted to investigate the impacts of SCPA on TIC and TIP. Third, to the best of our knowledge again, limited research has looked into the impacts of s-commerce experiences on SCI and e-WOMI, which in our case is tested as a control variable and found to be significant. This study also suggests a new viewpoint for practitioners in understanding how to design an effective s-commerce community and how consumers' relationships within such a community are initially established based on SCCA and SCPA. The results of this study advocate that community and platform attributes must be carefully taken, because both attributes carry different insights for any form of trusting mechanism. 
This study also presents in-depth managerial implications to e-vendors and service providers. First, the results affirm that SCCA create trust, which then improves both SCI and e-WOMI. While the results partially complement past studies' insights (Zhang et al., 2014), they also add to the existing literature by empirically proving that both SCCA and SCPA convincingly add to consumers' behavioral intents. Firms could encompass these SCCA into their business models and engage themselves with their consumers for further improvement of trust. Second, the results also indicate that firms that are already exercising s-commerce in their business models should enhance the applicability of SCPA, because firms with less social media platform attributes earn low customer engagement and market share versus firms with innovative social media platform attributes such as Amazon, Jingdong, and many more. Practitioners can enhance PI by making more realtime, less hassled, and electronically richer interactions between consumers by adding new social media online tools to their platforms. Third, for an increase in PS in the scommerce atmosphere, managers should provide user-friendly and convenient channels of communication for community members to build and strengthen relationships. Fourth, SCPA can also help service providers by establishing online dashboards to address consumers' interactivity and personalization concerns. The establishment of such dashboards will enhance consumers' interactivity and personalization in the perspective of product delivery, information access, and online transaction tracing mechanism. This exercise will engage more consumers towards the vendor's products and services, upon which the practitioners will enjoy customer participation in product and service improvement. Fifth, to encourage consumer participation and socialization, practitioners could incentivize consumers with social tools such as virtual gifts and social games. Finally, upon mapping consumers' clickstreams at different time interval visits, practitioners could also personalize the information for consumers' shopping specificity and browsing goals. This strategy could further top up PP.

\subsection{Limitations and future research directions}

Before adopting the generalized concept of the study, the findings should be considered under the following limitations. First, this study focuses on the mediating role of trust in the relationship between SCCA and SCPA and SCI and e-WOMI. There could also be other mediators such as usefulness, convenience, etc. that could be exciting future research directions. Second, as a large population of the respondents in our study is Chinese, it makes the study more region specific. Future studies can vary the respondent set by 
capturing responses from different geographical locations, which can help predict new dimensions in selected sets of variables. Third, our study's SCCA and SCPA are limited to our choice. Thus, future studies could explore cutting-edge technologies used nowadays over the Internet as other platform attributes. Fourth and lastly, more control variables such as the disposable income of respondents for shopping could also be a promising research direction for future in-depth insights into the s-commerce perspective.

\section{Reference}

Akram, M. S., Malik, A., Akhter, M., \& Shakir, M. A. (2019). Exploring the interrelationships between technological predictors and behavioral mediators in online tax filing : The moderating role of perceived risk. Government Information Quarterly, 1-15. https://doi.org/10.1016/j.giq.2018.12.007

Anderson, J. C., \& Gerbing, D. W. (1988). Structural equation modeling in practice: A review and recommended two-step approach. Psychological Bulletin, 103(3), 411423. https://doi.org/10.1037/0033-2909.103.3.411

Baethge, C., Klier, J., \& Klier, M. (2016). Social commerce-state-of-the-art and future research directions. Electronic Markets, 26(3), 269-290.

https://doi.org/10.1007/s12525-016-0225-2

Bhattacherjee, A. (2001). An empirical analysis of the antecedents of electronic commerce service continuance. Decision Support Systems, 32(2), 201-214. https://doi.org/10.1016/S0167-9236(01)00111-7

Busalim, A. H., \& Hussin, A. R. C. (2016). Understanding social commerce: A systematic literature review and directions for further research. International Journal of Information Management, 36(6), 1075-1088. https://doi.org/10.1016/j.ijinfomgt.2016.06.005

Chakraborty, R., Lee, J., Bagchi-Sen, S., Upadhyaya, S., \& Raghav Rao, H. (2016). Online shopping intention in the context of data breach in online retail stores: An examination of older and younger adults. Decision Support Systems, 83, 47-56. https://doi.org/10.1016/j.dss.2015.12.007

Chang, S. E., Liu, A. Y., \& Shen, W. C. (2017). User trust in social networking services: A comparison of Facebook and LinkedIn. Computers in Human Behavior, 69, 207-217. https://doi.org/10.1016/j.chb.2016.12.013

Chen, A., Lu, Y., \& Wang, B. (2017). Customers' purchase decision-making process in 
social commerce: A social learning perspective. International Journal of Information Management, 37(6), 627-638. https://doi.org/10.1016/j.ijinfomgt.2017.05.001

Chen, J., \& Shen, X. L. (2015). Consumers' decisions in social commerce context: An empirical investigation. Decision Support Systems, 79, 55-64. https://doi.org/10.1016/j.dss.2015.07.012

Chen, J. V., Su, B. C., \& Widjaja, A. E. (2016). Facebook C2C social commerce: A study of online impulse buying. Decision Support Systems, 83, 57-69. https://doi.org/10.1016/j.dss.2015.12.008

Choi, Y. K., Seo, Y., \& Yoon, S. (2017). E-WOM messaging on social media. Internet Research, 27(3), 495-505. https://doi.org/10.1108/IntR-07-2016-0198

Connelly, B. L., Crook, T. R., Combs, J. G., Ketchen, D. J., \& Aguinis, H. (2018). Competenceand Integrity-Based Trust in Interorganizational Relationships: Which Matters More? Journal of Management, 44(3), 919-945. https://doi.org/10.1177/0149206315596813

Dholakia, U. M., \& Talukdar, D. (2004). How social influence affects consumption trends in emerging markets: An empirical investigation of the consumption convergence hypothesis. Psychology and Marketing, 21(10), 775-797. https://doi.org/10.1002/mar.20029

Farivar, S., Turel, O., \& Yuan, Y. (2017). A trust-risk perspective on social commerce use: an examination of the biasing role of habit. Internet Research, 27(3), 586-607. https://doi.org/10.1108/IntR-06-2016-0175

Fornell, C., \& Larcker, D. (1981). Evaluating Structural Equation Models with Unobservable Variables and Measurement Error. Journal of Marketing Research, $18(1), 50,39$.

Gavilan, D., Avello, M., \& Martinez-Navarro, G. (2018). The influence of online ratings and reviews on hotel booking consideration. Tourism Management, 66, 53-61. https://doi.org/10.1016/j.tourman.2017.10.018

Gefen, D., \& Straub, D. W. (2003). Managing User Trust in B2C e-Services. E-Service Journal, 2(2), 7-24. https://doi.org/10.1353/esj.2003.0011

Gerbing, D. W., \& Anderson, J. C. (1988). An Updated Paradigm for Scale Development Incorporating Unidimensionality and Its Assessment. Journal of Marketing Research, 25(2), 186. https://doi.org/10.2307/3172650

Gibreel, O., AlOtaibi, D. A., \& Altmann, J. (2018). Social commerce development in 
emerging markets. Electronic Commerce Research and Applications, 27, 152-162. https://doi.org/10.1016/J.ELERAP.2017.12.008

Hair, J. F., Ringle, C. M., \& Sarstedt, M. (2011). PLS-SEM: Indeed a Silver Bullet. The Journal of Marketing Theory and Practice, 19(2), 139-152. https://doi.org/10.2753/MTP1069-6679190202

Hajli, M. (2013). A research framework for social commerce adoption. Information Management \& Computer Security, 21(3), 144-154. https://doi.org/10.1108/IMCS04-2012-0024

Hajli, M. N. (2014). The role of social support on relationship quality and social commerce. Technological Forecasting and Social Change, 87, 17-27. https://doi.org/10.1016/j.techfore.2014.05.012

Hajli, N. (2015). Social commerce constructs and consumer's intention to buy. International Journal of Information Management, 35(2), 183-191. https://doi.org/10.1016/j.ijinfomgt.2014.12.005

Hajli, N., Lin, X., Featherman, M., \& Wang, Y. (2015). Social Word of Mouth: How Trust Develops in the Market. International Journal of Market Researchch, 56(5), 673689.

Hajli, N., Sims, J., Zadeh, A. H., \& Richard, M. O. (2017). A social commerce investigation of the role of trust in a social networking site on purchase intentions. Journal of Business Research, 71, 133-141. https://doi.org/10.1016/j.jbusres.2016.10.004

Hajli, N., Wang, Y., Tajvidi, M., \& Hajli, M. S. (2017). People, Technologies, and Organizations Interactions in a Social Commerce Era. IEEE Transactions on Engineering Management, 64(4), 594-604. https://doi.org/10.1109/TEM.2017.2711042

Hsu, P.-Y., Lei, H.-T., Huang, S.-H., Liao, T. H., Lo, Y.-C., \& Lo, C.-C. (2018). Effects of sentiment on recommendations in social network. Electronic Markets, 1-10. https://doi.org/10.1007/s12525-018-0314-5

Hu, L., \& Bentler, P. M. (1999). Cutoff criteria for fit indexes in covariance structure analysis: conventional criteria versus New alternatives. Structural Equation Modeling, 6(1), 1-55.

Hu, M., Zhang, M., \& Luo, N. (2016). Understanding participation on video sharing communities: The role of self-construal and community interactivity. Computers in Human Behavior, 62, 105-115. https://doi.org/10.1016/j.chb.2016.03.077 
Huang, Z., \& Benyoucef, M. (2013). From e-commerce to social commerce: A close look at design features. Electronic Commerce Research and Applications, 12(4), 246-259. https://doi.org/10.1016/J.ELERAP.2012.12.003

Huang, Z., \& Benyoucef, M. (2017). The effects of social commerce design on consumer purchase decision-making: An empirical study. Electronic Commerce Research and Applications, 25, 40-58. https://doi.org/10.1016/J.ELERAP.2017.08.003

Kang, J.-Y. M., \& Johnson, K. K. P. (2015). F-Commerce platform for apparel online social shopping: Testing a Mowen's 3M model. International Journal of Information Management, 35(6), 691-701. https://doi.org/10.1016/J.IJINFOMGT.2015.07.004

Kim, H.-W., Chan, H. C., \& Kankanhalli, A. (2012). What Motivates People to Purchase Digital Items on Virtual Community Websites? The Desire for Online SelfPresentation. Information Systems Research, 23(4), 1232-1245. https://doi.org/10.1287/isre.1110.0411

Kim, N., \& Kim, W. (2018). Do your social media lead you to make social deal purchases? Consumer-generated social referrals for sales via social commerce. International Journal of Information Management, 39, 38-48. https://doi.org/10.1016/J.IJINFOMGT.2017.10.006

Kim, S., \& Park, H. (2013). Effects of various characteristics of social commerce (scommerce) on consumers' trust and trust performance. International Journal of Information Management, 33(2), 318-332. https://doi.org/10.1016/j.ijinfomgt.2012.11.006

Kline, R. B. (2010). Principles and Practice of Structural Equation Modeling (Third Edit). The Guilford Press.

Li, C.-Y. (2017). How social commerce constructs influence customers' social shopping intention? An empirical study of a social commerce website. Technological Forecasting and Social Change. https://doi.org/https://doi.org/10.1016/j.techfore.2017.11.026

Li, C.-Y. Y., \& Ku, Y.-C. C. (2018). The power of a thumbs-up: Will e-commerce switch to social commerce? Information and Management, 55(3), 340-357. https://doi.org/10.1016/j.im.2017.09.001

Liang, T.-P. P., Ho, Y.-T. T., Li, Y.-W. W., \& Turban, E. (2011). What Drives Social Commerce: The Role of Social Support and Relationship Quality. International Journal of Electronic Commerce, 16(2), 69-90. https://doi.org/10.2753/JEC1086- 


\section{4}

Liang, T.-P., \& Turban, E. (2011). Introduction to the Special Issue Social Commerce: A Research Framework for Social Commerce. International Journal of Electronic Commerce, 16(2), 5-14. https://doi.org/10.2753/JEC1086-4415160201

Lin, J., Yan, Y., Chen, S., \& Luo, X. (2017). Understanding the Impact of Social Commerce Website Technical Features on Repurchase Intention: A Chinese Guanxi Perspective. Journal of Electronic Commerce Research, 18(3).

Lin, X., Li, Y., \& Wang, X. (2017). Social commerce research: Definition , research themes and the trends. International Journal of Information Management, 37(3), 190-201. https://doi.org/10.1016/j.ijinfomgt.2016.06.006

Ng, C. S. P. (2013). Intention to purchase on social commerce websites across cultures: A cross-regional study. Information and Management, 50(8), 609-620. https://doi.org/10.1016/j.im.2013.08.002

Nunnally, J. C. (1978). Psychometric Theory (2nd ed.). New York: Mcgraw-Hill College. Ortiz, J., Chih, W.-H., \& Teng, H.-C. (2017). Electronic word of mouth in the Taiwanese social networking community: participation factors. Internet Research, 27(5), 1058-1084. https://doi.org/10.1108/IntR-09-2016-0276

Podsakoff, P. M., MacKenzie, S. B., Lee, J.-Y., \& Podsakoff, N. P. (2003). Common method biases in behavioral research: a critical review of the literature and recommended remedies. The Journal of Applied Psychology, 88(5), 879-903. https://doi.org/10.1037/0021-9010.88.5.879

Ridings, C. M., Gefen, D., \& Arinze, B. (2002). Some antecedents and effects of trust in virtual communities. The Journal of Strategic Information Systems, 11(3-4), 271295. https://doi.org/10.1016/S0963-8687(02)00021-5

Shanmugam, M., Sun, S., Amidi, A., Khani, F. F., \& Khani, F. F. (2016). The applications of social commerce constructs. International Journal of Information Management, 36(3), 425-432. https://doi.org/10.1016/j.ijinfomgt.2016.01.007

Shareef, M. A., Kumar, V., Kumar, U., \& Dwivedi, Y. K. (2011). E-Government Adoption Model (GAM): Differing service maturity levels. Government Information Quarterly, 28(1), 17-35. https://doi.org/10.1016/j.giq.2010.05.006

Sharma, S., Menard, P., \& Mutchler, L. A. (2017). Who to Trust? Applying Trust to Social Commerce. Journal of Computer Information Systems, 00(00), 1-11. https://doi.org/10.1080/08874417.2017.1289356 
Sheikh, Z., Islam, T., Rana, S., Hameed, Z., \& Saeed, U. (2017). Acceptance of social commerce framework in Saudi Arabia. Telematics and Informatics, 34(8), 16931708. https://doi.org/10.1016/J.TELE.2017.08.003

Shen, X.-L., Lee, M. K. O., \& Cheung, C. M. K. (2014). Exploring online social behavior in crowdsourcing communities: A relationship management perspective. Computers in Human Behavior, 40, 144-151. https://doi.org/10.1016/J.CHB.2014.08.006

Shen, X. L., Li, Y. J., Sun, Y., Chen, Z., \& Wang, F. (2018). Understanding the role of technology attractiveness in promoting social commerce engagement: Moderating effect of personal interest. Information and Management, (July 2017), 0-1. https://doi.org/10.1016/j.im.2018.09.006

Stewart, K. J. (2003). Trust Transfer on the World Wide Web. Organization Science, 14(1), 5-17. https://doi.org/10.1287/orsc.14.1.5.12810

Tajvidi, M., Wang, Y., Hajli, N., \& Love, P. E. D. (2017). Brand value Co-creation in social commerce: The role of interactivity, social support, and relationship quality. Computers in Human Behavior. https://doi.org/10.1016/j.chb.2017.11.006

Thapliyal, H. (2018). Internet of Things-Based Consumer Electronics: Reviewing Existing Consumer Electronic Devices, Systems, and Platforms and Exploring New Research Paradigms. IEEE Consumer Electronics Magazine, 7(1), 66-67. https://doi.org/10.1109/MCE.2017.2755219

Um, N. (2018). Antecedents and Consequences of Consumers ' Attitude toward Social Commerce Sites, 6491. https://doi.org/10.1080/10496491.2018.1448324

Xu, B., Jones, D. R., \& Shao, B. (2009). Volunteers' involvement in online community based software development. Information \& Management, 46(3), 151-158. https://doi.org/10.1016/J.IM.2008.12.005

Yahia, I. Ben, Al-Neama, N., \& Kerbache, L. (2018). Investigating the drivers for social commerce in social media platforms: Importance of trust, social support and the platform perceived usage. Journal of Retailing and Consumer Services, 41, 11-19. https://doi.org/10.1016/J.JRETCONSER.2017.10.021

Ye, Q., Law, R., Gu, B., \& Chen, W. (2011). The influence of user-generated content on traveler behavior: An empirical investigation on the effects of e-word-of-mouth to hotel online bookings. Computers in Human Behavior, 27(2), 634-639. https://doi.org/10.1016/j.chb.2010.04.014

Yu, C.-H., Tsai, C.-C., Wang, Y., Lai, K.-K., \& Tajvidi, M. (2018). Towards building a value 
co-creation circle in social commerce. Computers in Human Behavior. https://doi.org/10.1016/J.CHB.2018.04.021

Zhang, H., Lu, Y., Gupta, S., \& Zhao, L. (2014). What motivates customers to participate in social commerce? the impact of technological environments and virtual customer experiences. Information and Management, 51(8), 1017-1030. https://doi.org/10.1016/j.im.2014.07.005

Zhang, K. Z. K., \& Benyoucef, M. (2016). Consumer behavior in social commerce: A literature review. Decision Support Systems. https://doi.org/10.1016/j.dss.2016.04.001

Zhou, L., Zhang, P., \& Zimmermannc, H.-D. (2013). Social commerce research: An integrated view. Electronic Commerce Research and Applications, 12(2), 61-68. https://doi.org/10.1016/J.ELERAP.2013.02.003

Zhu, J., Goraya, M., Cai, Y., Zhu, J., Goraya, M. A. S., \& Cai, Y. (2018). Retailer-Consumer Sustainable Business Environment: How Consumers' Perceived Benefits Are Translated by the Addition of New Retail Channels. Sustainability, 10(9), 1-22. https://doi.org/10.3390/SU10092959

\section{Appendix A. Constructs and Measurement Items}

Recommendation and referrals adapted from Chen et al. (2017), Hajli (2015) and Li (2017)

ReRf1: The recommendations and referrals I receive from the holiday booking website community are valuable.

ReRf2: The recommendations and referrals I receive from the holiday booking website community are informative.

ReRf3: Overall, the information I receive from the holiday booking website community is helpful.

Forums and communities adapted from Chen et al. (2017) and Hajli (2015)

FC1: When faced with difficulties, community on holiday booking website is helpful. FC2: When encountering a problem, community on holiday booking website is supportive.

FC3: Overall, Forums, and communities of holiday booking website are caring.

Rating and reviews adapted from Chen et al. (2017), Hajli (2015) and Li (2017)

RR1: The ratings and reviews provided on holiday booking website by the community are factual.

RR2: The ratings and reviews provided on holiday booking website by the community are accurate.

RR3: Overall, the ratings and reviews provided on holiday booking website by the community are credible. 


\section{Perceived Interactivity adapted from Zhang et al. (2014)}

PI1: The tools provided by holiday booking website allow me to update content that exists in the online medium.

PI2: The tools provided by holiday booking website allow me to create content as per my imagination.

PI3: Holiday booking website allows me to change or influence the way the online medium looks.

\section{Perceived Personalization adapted from Zhang et al. (2014)}

PP1: Holiday booking website understands my specific needs.

PP2: Holiday booking website knows what I want.

PP3: Holiday booking website stores all my preferences and offers me extra services based on my preferences.

PP4: Holiday booking website does a pretty good job guessing what kinds of things I might want and making suggestions.

\section{Perceived Sociability adapted from Zhang et al. (2014)}

PS1: Holiday booking website enables me to get a good impression of other customers in the environment.

PS2: Holiday booking website enables me to develop good social relationships with other customers in the environment.

PS3: Holiday booking website enables me to feel part of the virtual community. PS4: Holiday booking website enables me to form close friendships with other customers in the environment.

Trust in platform adapted from Gefen \& Straub (2003) and Yahia et al. (2018)

TIP1: Promises made by holiday booking websites are likely to be reliable.

TIP2: I do not doubt the honesty of holiday booking sites.

TIP3: I expect that the advice given on holiday booking sites is their best judgment.

TIP4: I believe holiday booking sites have my information safety in their minds.

Trust in community adapted from Ridings et al. (2002) and Yahia et al. (2018)

TIC1: I feel very confident about the skills that the other members of the holiday booking website's community have in relation to the topics we discuss.

TIC2: The other participants on the holiday booking website's Community have much knowledge about the subject we discuss.

TIC3: The participants in the holiday booking website's Community will do everything within their capacity to help others.

TIC4: The participants in the holiday booking website's Community are concerned about what is important to others.

S-commerce Intentions adapted from Chen and Shen (2015) and Liang et al. (2011)

SCI1: I will consider the shopping experiences of other members on the holiday booking website when I want to book/buy holiday tickets/places.

SCI2: I will ask other members on the holiday website to provide me with their suggestions before I book/buy holiday tickets/places.

SCI3: I am willing to buy the products recommended by other members on the holiday booking websites. 


\section{E-WOM Intentions adapted from Chen and Shen (2015) and Liang et al. (2011)}

E-WOMI1: I am willing to provide my experiences and suggestions when other members of the holiday website want my advice on booking holidays.

E-WOMI2: I am willing to share my own holiday booking experience with other members on the holiday website.

E-WOMI3: I am willing to recommend a product that is worth buying to other members on the holiday website.

Note: $R R=$ Ratings and reviews, ReRf=Recommendation and referrals, FC=Forums and communities, $P I=P e r c e i v e d$ interactivity, $P P=$ Perceived personalization, $P S=$ Perceived sociability, TIC=Trust in community, TIP=Trust in platform, $S C I=S$-commerce intention, $E$ WOMI $=e-$ WOM intention. 